\title{
A Tractable Model for Indices Approximating the Growth Optimal Portfolio
}

This version: 12 November 2012

\author{
Jan Baldeaux $^{\mathrm{a}}$, Katja Ignatieva ${ }^{\mathrm{b}}$, Eckhard Platen ${ }^{\mathrm{c}}$ \\ ${ }^{a}$ Finance Discipline Group, University of Technology Sydney, Australia. \\ E-mail: jan.baldeaux@uts.edu.au \\ ${ }^{\mathrm{b}}$ School of Risk and Actuarial Studies, Australian School of Business, University \\ of New South Wales, Sydney, Australia.E-mail: k.ignatieva@unsw.edu.au \\ ${ }^{\mathrm{c}}$ Finance Discipline Group and School of Mathematical Studies, University of \\ Technology Sydney, Australia. E-mail: eckhard.platen@uts.edu.au
}

\begin{abstract}
The growth optimal portfolio (GOP) plays an important role in finance, where it serves as the numéraire portfolio, with respect to which contingent claims can be priced under the real world probability measure. This paper models the GOP using a time dependent constant elasticity of variance (TCEV) model. The TCEV model has high tractability for a range of derivative prices and fits well the dynamics of a global diversified world equity index. This is confirmed when pricing and hedging various derivatives using this index.
\end{abstract}

Key words: growth optimal portfolio, constant elasticity of variance model, kernel estimation, diffusion coefficient function, derivative hedging 


\section{Introduction}

The growth optimal portfolio (GOP) plays an important role in the finance literature. It has been studied in, e.g. Kelly (1956), see also Latané (1959), Breiman (1960), Thorp (1961), Markowitz (1976), Long (1990) and most recently MacLean et al. (2011). It is the portfolio which maximizes expected log-utility from terminal wealth. Furthermore, the GOP plays a crucial role under the benchmark approach, which is a unifying framework for portfolio optimization, risk management, and derivative pricing, see Platen and Heath (2010). In particular, the GOP can be employed as numéraire portfolio and contingent claims can, thus, be priced under the real world probability measure, see Platen and Heath (2010) and Becherer (2001) for references on the numéraire portfolio in a semimartingale setting. This avoids the assumption on the existence of an equivalent risk neutral probability measure and allows for a much richer modeling world than the classical risk neutral approach can provide. It has been shown in Platen (2005) and Platen and Rendek (2011) via diversification theorems that an equi-weighted portfolio represents, under rather general assumptions, a good proxy for the GOP. Such a proxy will be employed in this paper.

Given the important role of the GOP, it is clear that one needs a good model for its dynamics. In Platen and Heath (2010), the minimal market model (MMM) has been used to model the GOP, which has performed reasonably well in empirical studies when investigating the ability of the GOP, in combination with other instruments, to replicate claims, see also Hulley and Platen (2012). The present paper will generalize the MMM, in the setting of the benchmark approach presented in Platen and Heath (2010), by developing a model which satisfies the following requirements:

- The model should be parsimonious, with each parameter having a clear interpretation.

- The discounted GOP should be modeled by a scalar diffusion driven by a single Brownian motion.

- The model should be estimated reliably from historical data.

- The model should admit explicit formulae for various derivatives and their hedge ratios including call and put options.

In particular, the above requirements postulate that the discounted GOP satisfies a scalar diffusion dynamics. The proposed specification will be reminiscent of the modified constant elasticity of variance model, see Cox (1975) and Heath and Platen (2002). Due to the time dependence of one of its parameters, we will refer to the proposed model as the time dependent CEV model, that is, the TCEV model. Under the TCEV model, we will recover the MMM, as well as other well known models.

We use non-parametric kernel-based estimation, a method that has enjoyed much attention in the statistics literature, see e.g. Florens-Zmirou (1993), Jacod (2000), Jiang and Knight (1997), Soulier (1998), and Stanton (1997), to estimate the diffusion coefficient function of the discounted GOP without assuming any particular scalar diffusion dynamics. It turns out that the diffusion coefficient function of the TCEV model fits surprisingly well the non-parametrically estimated diffusion coefficient function. Finally, the paper contributes to the existing literature on CEV type models by providing explicit pricing formulae for savings account bonds, call and put 
options, as well as their hedge ratios. The novel results allow us to compare conveniently the ability of the MMM and the TCEV model to hedge various derivatives. We identify by how much the TCEV model outperforms its competitor in the sense that it can replicate the same payoff at smaller initial cost when facing comparable hedge errors.

The structure of the paper is as follows: In Section 2, we recall key properties of the GOP, specify models of the GOP that have appeared in the literature, and discuss the TCEV model. Non-parametric kernel-based estimation, which is used to parameterize the TCEV model, is discussed in Section 3. Consequently, we apply the techniques discussed in Section 3 to estimate the parameters of the TCEV model in Section 4, where we approximate the GOP using an equi-weighted index, the EWI114. In Section 5 , we derive closed-form solutions for various derivatives and their hedge ratios under the TCEV model. The results are applied in Section 6 to pricing and hedging using the parameter estimates from Section 4. Finally, Section 7 concludes the paper. All proofs are presented in the Appendix.

\section{Modeling the GOP}

In this section, we discuss general properties of the GOP, recall well-known models of the GOP from the literature and discuss how we intend to generalize these existing models.

We work on a filtered probability space $(\Omega, \mathcal{A}, \underline{\mathcal{A}}, P)$ with the filtration $\underline{\mathcal{A}}=\left(\mathcal{A}_{t}\right)_{t \geq 0}$ satisfying the usual conditions, see Karatzas and Shreve (1991). In our study we employ only one source of uncertainty given by a standard Wiener process $W=$ $\left\{W_{t}, t \geq 0\right\}$. In a continuous time financial market, as described in Platen and Heath (2010), the savings account discounted GOP $\bar{S}_{t}^{*}$ satisfies the stochastic differential equation (SDE):

$$
d \bar{S}_{t}^{*}=\bar{S}_{t}^{*} \theta_{t}\left(\theta_{t} d t+d W_{t}\right)
$$

for $t \geq 0$, see equation (10.2.8) in Platen and Heath (2010). Here, $\bar{S}_{0}^{*}>0$ is the initial value and $\theta_{t}$ denotes the volatility of the GOP or market price of risk. We are also interested in the benchmarked savings account, $\hat{B}_{t}=\frac{1}{S_{t}^{*}}$, whose dynamics are given by the SDE

$$
d \hat{B}_{t}=-\theta_{t} \hat{B}_{t} d W_{t}
$$

that is, the process $\hat{B}=\left\{\hat{B}_{t}, t \geq 0\right\}$ is a local martingale. As will be shown in Section 5 , see in particular equations (5.1) and (5.2), we can hedge in our complete two-asset market contingent claims using the savings account and the GOP. Hence in order to hedge, we only need to specify $\theta_{t}$, which determines the diffusion coefficient of the local martingale $\hat{B}_{t}$.

We now discuss a particular family of scalar diffusion models for the discounted GOP, where most of these models have previously appeared in the literature. One can call this class of models the class of local volatility function models, see Dupire (1994) and Derman and Kani (1994). More precisely, we assume that the GOP volatility, or 
market price of risk, is of the form

$$
\theta_{t}=g\left(\frac{\bar{S}_{t}^{*}}{\alpha_{t}}\right)
$$

where $g(\cdot)$ is a given function and $\alpha_{t}$ is a deterministic function of time, which is growing on average like the discounted GOP. This leads to the following SDE for the discounted GOP:

$$
d \bar{S}_{t}^{*}=\bar{S}_{t}^{*} g\left(\frac{\bar{S}_{t}^{*}}{\alpha_{t}}\right)^{2} d t+\bar{S}_{t}^{*} g\left(\frac{\bar{S}_{t}^{*}}{\alpha_{t}}\right) d W_{t}
$$

Intuitively, $\frac{\bar{S}_{t}^{*}}{\alpha_{t}}$ can be expected to form, under an appropriate choice of $\alpha_{t}$, a process that has a stationary probability density. The local volatility function model (2.4) yields different model specifications discussed in the literature:

- The Black-Scholes (BS) model, see Black and Scholes (1973), is the standard market model and highly tractable. It assumes that the volatility $\theta_{t}$ of the GOP is constant. By setting $g\left(\frac{\bar{S}_{t}^{*}}{\alpha_{t}}\right)=\sigma$, its dynamics is recovered as a special case.

- The constant elasticity of variance (CEV) model, see Cox (1975) and Schroder (1989), has been modified in Heath and Platen (2002) to model the discounted GOP, such that

$$
g\left(\frac{\bar{S}_{t}^{*}}{\alpha}\right)=\left(\frac{\bar{S}_{t}^{*}}{\alpha}\right)^{a-1},
$$

where $a \in(-\infty, 1)$ and $\alpha$ is constant, yielding the modified constant elasticity of variance (MCEV) model.

- The minimal market model (MMM), which is highly tractable, has previously been discussed in Platen (2001, 2002). In this case

$$
\alpha_{t}=\alpha_{0} \exp \{\eta t\}
$$

for $t \geq 0$. Here $\alpha_{0}>0$ is some initial value and $\eta>0$ is the net growth rate. The volatility $\theta_{t}$, which satisfies in this case the equation

$$
g\left(\frac{\bar{S}_{t}^{*}}{\alpha_{t}}\right)=\sqrt{\frac{\alpha_{t}}{\bar{S}_{t}^{*}}}=\left(\frac{\bar{S}_{t}^{*}}{\alpha_{t}}\right)^{a-1},
$$

with $a=1 / 2$, allows us to model the leverage effect, that is, as $\bar{S}_{t}^{*}$ decreases, the volatility increases and vice versa. Furthermore, the volatility can be shown to have a stationary probability density.

- The proposed time dependent constant elasticity of variance (TCEV) model has the volatility

$$
g\left(\frac{\bar{S}_{t}^{*}}{\alpha_{t}}\right)=c\left(\frac{\bar{S}_{t}^{*}}{\alpha_{t}}\right)^{a-1}
$$

for $c>0, a \in(-\infty, 1)$, and $\alpha_{t}$, defined as in (2.6), is an exponential function of time. When $\alpha_{t}$ growths with the average growth rate of $\bar{S}_{t}^{*}$, then the volatility has a stationary probability density.

Within this paper we regard the tractability of a model as paramount, and aim to 
identify a highly tractable model. Therefore, it is reasonable to aim for the modeling of the squared volatility by a time homogeneous, nonnegative scalar diffusion process, which has a stationary probability density. However, tractable scalar diffusions are rare. Affine diffusions are the best known class of such processes. We remark that any affine diffusion process, which is a time homogeneous, nonnegative diffusion, is necessarily of square root type, see Brown and Schaefer (1994a,b), and Duffie and Gârleanu (2001). We will now show for the TCEV model that its volatility is a function of a square root process:

Assuming that

$$
g\left(\frac{\bar{S}_{t}^{*}}{\alpha_{t}}\right)=c\left(\frac{\bar{S}_{t}^{*}}{\alpha_{t}}\right)^{a-1}
$$

where $\alpha_{t}$ satisfies $(2.6), c>0$ and $a \in(-\infty, 1)$, leads by (2.4) to the SDE

$$
d \bar{S}_{t}^{*}=c^{2} \alpha_{t}^{2-2 a}\left(\bar{S}_{t}^{*}\right)^{2 a-1} d t+c \alpha_{t}^{1-a}\left(\bar{S}_{t}^{*}\right)^{a} d W_{t} .
$$

Furthermore, we introduce the time homogenous diffusion process $Y=\left\{Y_{t}, t \geq 0\right\}$ with the SDE

$$
d Y_{t}=\left(c^{2} Y_{t}^{2 a-1}-\eta Y_{t}\right) d t+c Y_{t}^{a} d W_{t}
$$

for $t \geq 0$ and $Y_{0}=y>0, c>0$ and exponent $a \in(-\infty, 1)$. The existence of a unique strong solution to equations (2.10) and (2.11) follows from Proposition 8.1. Using the SDE (2.11), one can verify that $\bar{S}_{t}^{*}$ can be represented as the product

$$
\bar{S}_{t}^{*}=\alpha_{t} Y_{t} .
$$

In (2.12), when multiplying $Y_{t}$ by $\alpha_{0}$, one might be tempted to conclude that $\alpha_{0}$ and $c$ play identical roles and thus, one should be omitted. However, within the TCEV model, the parameter $c$ determines the level of the volatility process, see (2.15) and $\alpha_{0}$ governs the level of the discounted GOP. It is evidenced in Section 4, where the model is fitted to the data, that both parameters are required since they serve different purposes.

It can be shown via the Itô formula that the process $X=\left\{X_{t}, t \geq 0\right\}$ given by $X_{t}=c^{-2} Y_{t}^{2(1-a)}$ satisfies the $\mathrm{SDE}$

$$
d X_{t}=\left((3-2 a)(1-a)-2(1-a) \eta X_{t}\right) d t+2(1-a) \sqrt{X_{t}} d W_{t},
$$

i.e. the process $Y=\left\{Y_{t}, t \geq 0\right\}$ is the power of a square-root process of dimension

$$
\nu=\frac{3-2 a}{1-a}
$$

where $\nu>2$ for $a \in(-\infty, 1)$. From Proposition 8.1 it follows that the process $\bar{S}_{t}^{*}$ satisfies the relation

$$
\bar{S}_{t}^{*} \stackrel{(d)}{=} Z_{\varphi(t)}^{\frac{1}{2-2 a}}
$$


where the equality holds in distribution and

$$
\varphi(t)=\frac{(1-a) \alpha_{0}^{2-2 a} c^{2}(\exp \{2(1-a) \eta t\}-1)}{2 \eta}
$$

and $Z=\left\{Z_{\varphi}, \varphi \geq 0\right\}$ is a squared Bessel process of dimension $\nu=\frac{3-2 a}{1-a}$.

From equation (2.9) and (2.12) it can be shown that the volatility equals

$$
\theta_{t}=g\left(\frac{\bar{S}_{t}^{*}}{\alpha_{t}}\right)=c Y_{t}^{(a-1)}=X_{t}^{-\frac{1}{2}}
$$

Therefore, the squared volatility $\theta_{t}^{2}=\frac{1}{X_{t}}$ satisfies the SDE

$$
d \theta_{t}^{2}=(1-a) \theta_{t}^{2}\left((1-2 a) \theta_{t}^{2}+2 \eta\right) d t-2(1-a)\left(\theta_{t}^{2}\right)^{\frac{3}{2}} d W_{t}
$$

which means that we have obtained a version of a $3 / 2$ - volatility model, see Heston (1997), Platen (1997) and Lewis (2000).

The representation (2.12) is useful for the estimation of diffusion coefficient functions as discussed below.

We conclude this section by mentioning that the process $\bar{S}^{*}=\left\{\bar{S}_{t}^{*}, t \geq 0\right\}$ does not become zero or $+\infty$ in finite time, which follows from the fact that a square root process of dimension $\nu>2$ has this property, see Revuz and Yor (1999).

\section{Nonparametric Estimation of Diffusion Coefficient Function}

It will be our aim to check empirically which diffusion coefficient function $g(\cdot)$ fits well historical data. Therefore, this section deals with the nonparametric kernel-based estimation of the diffusion coefficient function in the SDE (2.10) of the discounted GOP. The above described TCEV model represents a special case in the class of local volatility function models admitted.

Starting with the work by Florens-Zmirou (1993), the kernel-based nonparametric estimation of diffusion coefficient functions has been discussed in several studies, see e.g. Soulier (1998) and Jacod (2000). For instance, Stanton (1997) and Jiang and Knight (1997) have applied a nonparametric kernel-based technique to estimate the drift and the diffusion coefficients of a short rate. Estimation of continuous-time diffusion processes which model the dynamics of a well diversified world stock index, constructed to provide a proxy for the GOP, has been discussed in Ignatieva and Platen (2012). The algorithms adopted from Ignatieva and Platen (2012) have been modified in order to allow for different model specifications considered in the present paper. 


\subsection{Kernel Density}

Given $n$ sample points $\left(x_{t_{1}}, \ldots, x_{t_{n}}\right)$, the kernel estimation density $\hat{f}\left(x, h_{n}\right)$ is defined by

$$
\hat{f}\left(x, h_{n}\right)=\frac{1}{n h_{n}} \sum_{l=1}^{n} K\left(\frac{x-x_{t_{l}}}{h_{n}}\right),
$$

where $K(\cdot)$ is a kernel function and $h_{n}$ is a bandwidth parameter controlling the degree of smoothness of the estimator. In this paper, we employ a Gaussian kernel function, i.e. we set

$$
K(z)=(2 \pi)^{-\frac{1}{2}} \exp \left\{-\frac{1}{2} z^{2}\right\}
$$

The kernel estimation density evaluated at point $x$ is estimated by the average of the kernel density values centered at the actual data points. The larger the distance between data point and estimated point, the lower is the contribution to the estimated density.

It has been shown in the literature, that the specific choice of the kernel $K(\cdot)$ does not affect much the performance of the resulting estimator. In fact, as argued in Epanechnikov (1969), any reasonable kernel gives almost optimal results. For the bandwidth $h_{n}$, one typically chooses the bandwidth which minimizes the asymptotic mean integrated squared error (MISE):

$$
\begin{aligned}
\operatorname{MiSE}\left\{\hat{f}\left(x, h_{n}\right)\right\} & =\mathbb{E} \int\left[\hat{f}\left(x, h_{n}\right)-f(x)\right]^{2} d x \\
& =\int(\underbrace{\mathbb{E}\left[\hat{f}\left(x, h_{n}\right)\right]-f(x)}_{\text {pointwise bias }})^{2} d x+\int \underbrace{\operatorname{Var}\left[\hat{f}\left(x, h_{n}\right)\right]}_{\text {pointwise variance }} d x,
\end{aligned}
$$

see, e.g., Wand and Jones (1995) and Botev et al. (2010). Here, $f(\cdot)$ denotes the true density underlying the data. The analysis simplifies when one considers AMISE, an asymptotic approximation of MISE, see Wand and Jones (1995). For $h_{n}=h$ such that $\lim _{n \rightarrow \infty} h_{n}^{2}=0$ and $\lim _{n \rightarrow \infty} n h_{n}=0$, and $f^{\prime \prime}(\cdot)$ being continuous and square-integrable, one can rewrite the pointwise bias and variance in equation (3.2) for $n \rightarrow \infty$ in terms of the Euclidean norm $\|\cdot\|$ on $\mathbb{R}$ as follows:

$$
\|\mathbb{E}[\hat{f}(x, h)]-f(x)\|^{2}=\frac{1}{4} h^{4}\left\|f^{\prime \prime}\right\|^{2}+o(h)
$$

and

$$
\int \operatorname{Var}[\hat{f}(x, h)] d x=\frac{1}{2 n h \sqrt{\pi}}+o\left((n h)^{-1}\right) .
$$

Minimizing AMISE, that is,

$$
\operatorname{AMISE}\{\hat{f}(x, h)\}=\frac{1}{4} h^{4}\left\|f^{\prime \prime}\right\|^{2}+\frac{1}{2 n h \sqrt{\pi}},
$$


yields an asymptotically optimal bandwidth

$$
h^{*}=\left(\frac{1}{2 n \sqrt{\pi}\left\|f^{\prime \prime} \mid\right\|^{2}}\right)^{1 / 5},
$$

which gives the minimal value

$$
\operatorname{AMISE}\left\{\hat{f}\left(x, h^{*}\right)\right\}=n^{-4 / 5} \frac{5\left\|f^{\prime \prime}\right\|^{2 / 5}}{4^{7 / 5} \pi^{2 / 5}} .
$$

The proof of the above results is provided in Wand and Jones (1995).

In a Gaussian framework, it is well-known that $\left\|f^{\prime \prime}\right\|$ only depends on the standard deviation of the data $\sigma$. The most popular data-driven bandwidth-selection technique, the plug-in method, suggests to replace the standard deviation by its sample estimate $\hat{\sigma}$. Consequently, starting with Silverman (1986) and Scott (1992), the literature suggests to choose the bandwidth based on the dispersion of its observations, $\hat{\sigma}$, and the number of sample points,

$$
h_{n}=h_{c} \hat{\sigma} n^{-\frac{1}{5}},
$$

where $h_{c} \in \mathbb{R}$ is a constant to be tuned. For the purpose of our analysis, the smoothness of the diffusion coefficient function estimate does not affect the parameter estimates from the fitted diffusion and thus we employ the standard 'rule-of-thumb' bandwidth, which suggests to set $h_{c}=1$, in combination with a Gaussian kernel.

\subsection{Diffusion Coefficient Function Estimator}

This section describes the estimation procedure for the diffusion coefficient function $\sigma\left(X_{t}\right)$ for the general scalar diffusion process $X=\left\{X_{t}, t \geq 0\right\}$ following the SDE

$$
d X_{t}=\mu\left(X_{t}\right) d t+\sigma\left(X_{t}\right) d W_{t} .
$$

Given the kernel estimation density in equation (3.1) with bandwidth determined in (3.8), we can calculate the first order estimator $\sigma_{1}^{2}(\cdot)$ of the squared diffusion coefficient function $\sigma^{2}(\cdot)$, as suggested by Stanton (1997), in the following way:

$$
\sigma_{1}^{2}\left(x_{t_{i-1}}\right)=\frac{1}{t_{i}-t_{i-1}} E\left(\left(x_{t_{i}}-x_{t_{i-1}}\right)^{2} \mid x_{t_{i-1}}=x\right)+O\left(t_{i}-t_{i-1}\right)
$$

using the approximation

$$
E\left(\left(x_{t_{i}}-x_{t_{i-1}}\right)^{2} \mid x_{t_{i-1}}=x\right) \approx \frac{\sum_{l=1}^{n}\left(x_{t_{l}}-x_{t_{l-1}}\right)^{2} K\left(\frac{x-x_{t_{l-1}}}{h}\right)}{\sum_{l=1}^{n} K\left(\frac{x-x_{t_{l-1}}}{h}\right)},
$$

where $t_{i}-t_{i-1}$ denotes the time step between successive observations. In addition to the above first order approximations, Stanton (1997) developed higher order approximations based on Taylor expansions. However, he showed that using approximations of higher order does not affect the order of convergence but may improve the approx- 


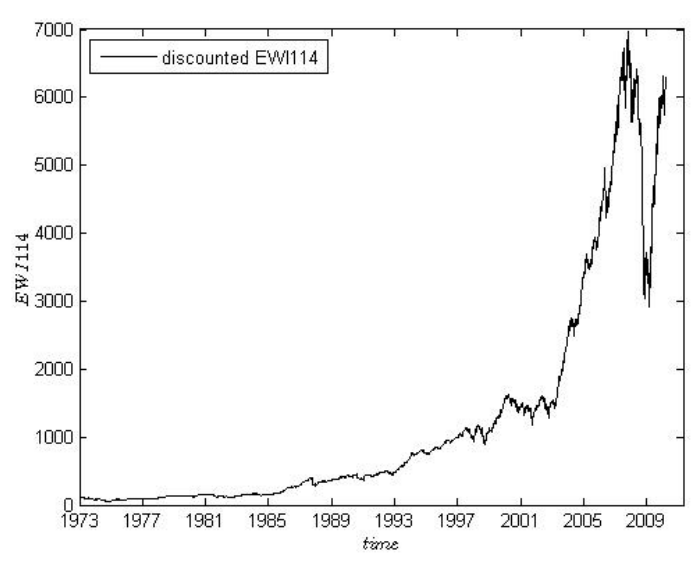

Fig. 1. Daily observed discounted EWI114 for the time period from January 1973 to July 2010 .

imations for a chosen time step $t_{i}-t_{i-1}$. For the purpose of our analysis we do not require higher order approximations to fit a parametric form to the estimated functions for the drift and diffusion coefficients and thus, the first order approximation (3.9) will be sufficient.

\section{Estimating the Diffusion Coefficient Function}

In this section we consider the equi-weighted index (EWI114), which uses 114 world industry sector indices as constituents provided by Datastream Thomson Financial, and was constructed in Platen and Rendek (2011) by taking 40 basis points proportional transaction costs into account. Thereby, we use daily data covering the time period from January 1973 to July 2010. The discounted EWI114 is plotted in Figure 1.

We have to estimate the parameters $\alpha_{0}$ and $\eta$ for the exponential function $\alpha_{t}$, which is given by

$$
\alpha_{t}=\alpha_{0} \exp \{\eta t\} .
$$

Two different estimation techniques are used for the MMM and TCEV respectively: When dealing with the MMM, it is convenient to recall the function $\varphi(t)$ from $(2.14)$ when setting $a=\frac{1}{2}$ and $c=1$,

$$
\varphi(t)=\frac{1}{4} \int_{0}^{t} \alpha_{s} d s=\frac{\alpha_{0}}{4 \eta}(\exp \{\eta t\}-1),
$$

which can be shown under the MMM to satisfy the equation

$$
\varphi(t)=\left[\sqrt{\bar{S}^{*}}\right]_{t},
$$

that is, when assuming that the process $\bar{S}^{*}$ is given by the stylized version of the MMM, see Platen and Heath (2010). Consequently, under the MMM we can fit $\varphi(t)$ 


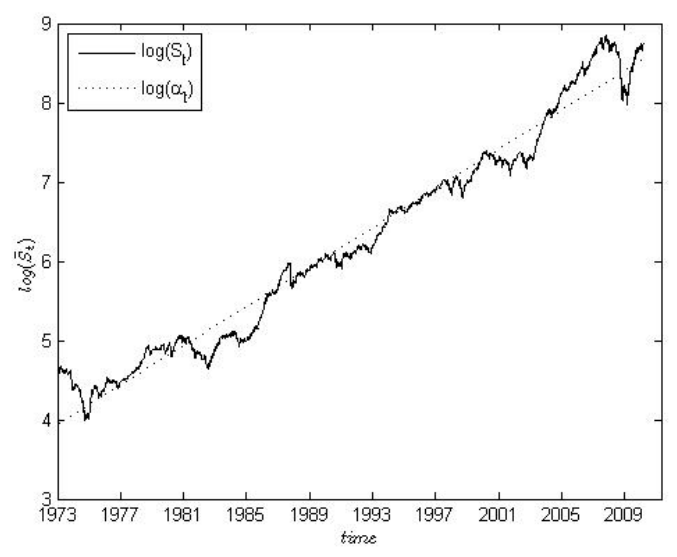

Fig. 2. Logarithm $\ln \left(\bar{S}_{t}^{*}\right)$ for the discounted EWI114 together with a fitted trend line of the form $\ln \left(\alpha_{t}\right)=\ln \left(\alpha_{0}\right)+\eta t$ for $\alpha_{0}=51.34$ and $\eta=0.1239$.

to the observed quadratic variation of $\sqrt{\bar{S}_{t}^{*}}$ to obtain the parameter values for $\alpha_{0}$ and $\eta$ using non-linear least squares estimation. The fitted parameter values under the MMM for the discounted EWI114, plotted in Figure 1, correspond to $\alpha_{0}=0.49$ and $\eta=0.1239$. We recall that for the MMM, the discounted GOP follows the dynamics of a time-changed squared Bessel process of dimension $\nu=4$ with $a=\frac{1}{2}$ and $c=1$. In this paper we do not restrict ourselves to the MMM and need to find other ways to estimate parameters.

The TCEV model does not allow us to estimate $\alpha_{0}$ and $\eta$ from the quadratic variation of $\sqrt{\bar{S}_{t}^{*}}$, since the latter depends on the parameter $a$, which is unknown at this stage. However, one can apply the non-linear least squares estimation to fit the logarithm $\ln \left(\alpha_{t}\right)$ to the logarithm $\ln \left(\bar{S}_{t}^{*}\right)$, as shown in Figure $2 .{ }^{1}$ The resulting parameter values are $\alpha_{0}=51.34$ and the same value $\eta=0.1239$ as in the special case of the MMM. We point out that the value $\alpha_{0}=51.34$ reflects the "average" level of the discounted GOP at the beginning of our dataset. Given the function $\alpha_{t}$, the normalized discounted GOP can be computed as $Y_{t}=\bar{S}_{t}^{*} / \alpha_{t}$, see (2.12). In the left panel of Figure 3, we plot the normalized process $Y$. We can now use this data to estimate the diffusion coefficient function in a non-parametric way as described in Section 3. The kernel density is estimated using a Gaussian kernel and a 'rule-of-thumb' bandwidth defined in (3.8). The resulting estimated approximation of the diffusion coefficient function is plotted in the right panel of Figure $3 .^{2}$ Fitting the estimated diffusion coefficient function to the functional form assumed for the diffusion coefficient function $\left(c Y^{a}\right)$

1 Proceeding in this way appears to be the most straightforward way to estimate $\alpha_{t}$, which is required to obtain a stationary process $Y_{t}=\bar{S}_{t}^{*} / \alpha_{t}$. We cannot apply the same methodology as in the case of the MMM (where parameters $a=\frac{1}{2}$ and $c=1$ are fixed). In our case the quadratic variation does not only depend on $\alpha_{t}$.

2 When estimating the diffusion coefficient function, we discard observations above the $90 \%$ quantile due to the fact that the probability distribution has a long right tail, indicating that there is not much data available to estimate reliably the diffusion coefficient function in the upper tail. Thus, we estimate the diffusion coefficient function from the truncated distribution. 

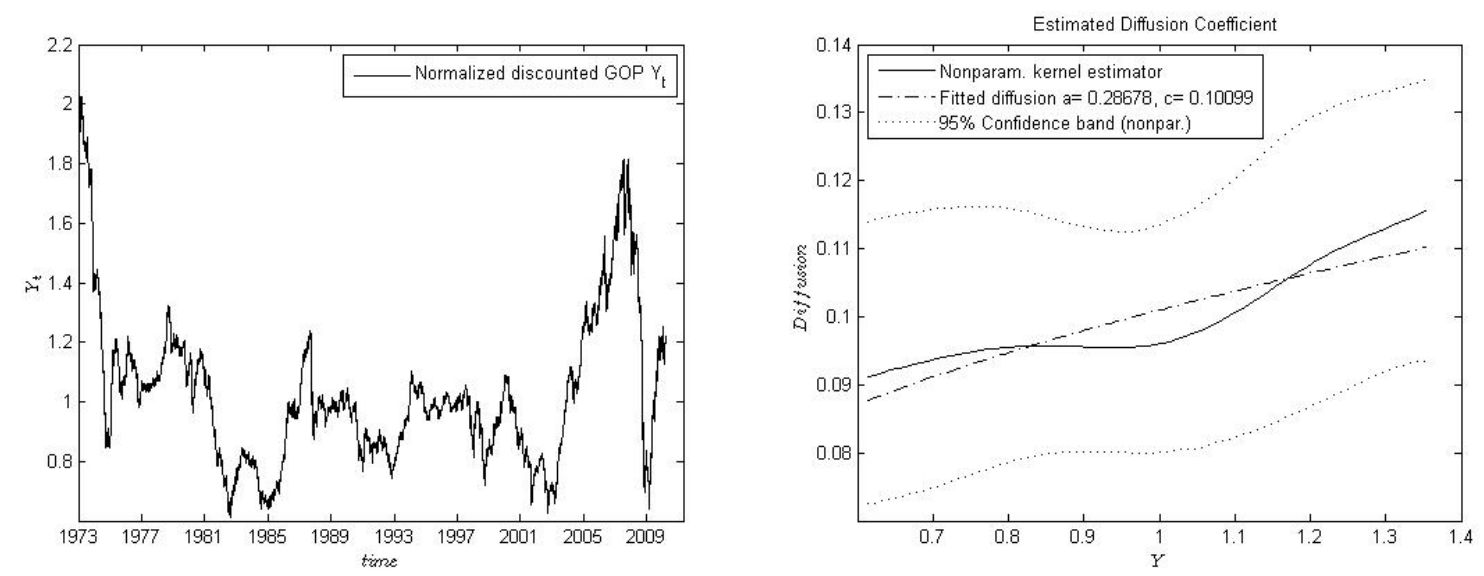

Fig. 3. Left panel: Normalized discounted GOP $Y_{t}=\bar{S}_{t}^{*} / \alpha_{t}$. Right panel: Estimated (solid line) and fitted (dashed-dotted line) diffusion coefficient function for the TCEV model specification with diffusion coefficient of the form $c Y^{a}$. Estimated parameter values for the first order approximation correspond to $c=0.1010$ and $a=0.2868$. Pointwise $95 \%$ confidence band for the diffusion coefficient function of $\tilde{Y}$. (dotted lines) is calculated using 10,000 iterations of the block bootstrap algorithm.

of the process $Y$, we obtain the parameter estimates $c=0.1010$ and $a=0.2868$. The fitted diffusion coefficient function is represented by the dashed-dotted line in the right panel of Figure 3. Note that by formula (2.13) the dimension of the embedded square root process corresponds to $\nu=3.4021$.

Along with the kernel-based diffusion estimator, and the fitted diffusion coefficient function, we plot a pointwise 95\% confidence band (dotted line), calculated using 10,000 iterations of the block bootstrap algorithm, see Künsch (1989). The idea to use blocks of observations rather than single observations in the bootstrap algorithm serves the purpose of preserving serial dependence in the data. The moving block bootstrap algorithm applied here, resamples the observed time series using approximately independent moving blocks. Thereby, it uses non-overlapping blocks of length $l$. For $n$ observations, we consider $k$ blocks of length $l(n=l k)$ constructed in the following way: Block one comprises observations from 1 to $l$, block two comprises observations from $l+1$ to $2 l$, etc. The last block $k$ comprises observations from $n-l+1$ to $n$. Block bootstrap estimation suggests to draw $k$ blocks with replacement from a set of blocks. Aligning these blocks in the order they were picked, gives us the bootstrap observations, which can then be used to compute standard errors and confidence bands.

\section{$5 \quad$ Real World Pricing under the TCEV Model}

In this section, we discuss the pricing and hedging of contingent claims under the model introduced in Section 2. Readers acquainted with risk-neutral derivative pricing would expect that we introduce an equivalent martingale measure (EMM) to price derivatives as done in Black and Scholes (1973), Merton (1973). However, the next proposition, which is derived in the Appendix, shows that this approach cannot be 
applied due to the lack of an EMM.

Proposition 5.1 Assume in our complete two-asset market model that under the real world measure, the discounted GOP satisfies $\bar{S}_{t}^{*}=\alpha_{t} Y_{t}$, where $\alpha_{t}$ and $Y_{t}$ are defined as in Section 2. Then an EMM does not exist.

Of course, our model violates the no free lunch with vanishing risk (NFLVR) condition, see Delbaen and Schachermayer (1994) and Delbaen and Schachermayer (1998). However, the model is free from those arbitrage opportunities which are economically meaningful, in the sense that they would allow to create strictly positive wealth out of zero initial wealth via a nonnegative portfolio, that is, under limited liability, see Loewenstein and Willard (2000) and Platen and Heath (2010). One can refer also to Fontana and Runggaldier (2012), Hulley and Schweizer (2010) and Kardaras (2010) who show that models similar to the TCEV model do not allow for strong forms of arbitrage even if an EMM fails to exist.

The observation in Proposition 5.1 leads us to apply real world pricing, which generalizes risk-neutral pricing, as shown in Platen and Heath (2010), and which is able to handle pricing and hedging for the model introduced above. In particular, we show how the benchmark approach finds the minimal price at which a contingent claim can be replicated. We replicate contingent claims by setting up a strategy $\delta=\left\{\delta_{t}=\left(\delta_{t}^{0}, \delta_{t}^{*}\right), t \geq 0\right\}$, which at time $t$ invests $\delta_{t}^{0}$ units in the savings account and $\delta_{t}^{*}$ units in the GOP, the benchmark. Consequently, the benchmarked value $\hat{S}_{t}^{\delta}$ of the corresponding portfolio at time $t$, that is its value in units of the GOP, is given by the sum

$$
\hat{S}_{t}^{\delta}=\delta_{t}^{0} \hat{B}_{t}+\delta_{t}^{*} .
$$

In particular, we are interested in portfolios which are self-financing, i.e. all changes in the portfolio value are only due to changes in asset values. This means, we have the SDE

$$
d \hat{S}_{t}^{\delta}=\delta_{t}^{0} d \hat{B}_{t} .
$$

Equation (5.2) reflects the conservation of value principle. Funds are neither added nor withdrawn from the portfolio. In the following, we only consider trading strategies which are self-financing.

The following proposition, see Theorem 10.3.1 in Platen and Heath (2010), sits at the heart of the benchmark approach.

Proposition 5.2 Each benchmarked nonnegative self-financing portfolio $\hat{S}_{t}^{\delta}$ forms a supermartingale, which means that

$$
\hat{S}_{t}^{\delta} \geq E_{t}\left(\hat{S}_{u}^{\delta}\right)
$$

for all $0 \leq t \leq u<\infty$

We point out that (5.3) illustrates that the GOP is the best performing portfolio in the following intuitive sense: When denominated in units of the GOP, portfolios trend downwards in the case of strict inequality or have no drift in the case of equality. It is clear that portfolios for which equality holds in (5.3) play a crucial role, hence we introduce the following notion. 
Definition 5.1 A portfolio is called fair, if the associated benchmarked portfolio value $\hat{S}_{t}^{\delta}$ forms a martingale, meaning that the current value is the best predictor of future values, that is,

for $0 \leq t \leq u<\infty$.

$$
\hat{S}_{t}^{\delta}=E_{t}\left(\hat{S}_{u}^{\delta}\right)
$$

It is our objective to replicate a given payoff at the minimal price. Combining Proposition 5.2 and Definition 5.1, it is clear that if we can find a fair portfolio which replicates the claim under consideration, then the set-up cost for this portfolio represents the minimal price at which the claim can be replicated. This is formalized in the Law of the Minimal Price:

Theorem 5.1 If a fair portfolio replicates a given nonnegative payoff at some future time, then this portfolio represents the minimal replicating portfolio among all nonnegative portfolios that replicate this payoff.

In the following, we derive fair portfolios and the associated price processes for several index derivatives under the benchmark approach. We emphasize that our model is complete, in the sense that for every nonnegative contingent claim there exists a fair strategy which perfectly replicates the claim. This follows immediately from Proposition 3.2. in Hulley and Platen (2012). Using (2.14), the following auxiliary notation is useful

$$
\begin{aligned}
\varphi(t) & =\frac{(1-a) \alpha_{0}^{2-2 a} c^{2}}{2 \eta}(\exp \{2(1-a) \eta t\}-1), \quad \Delta \varphi(t)=\varphi(T)-\varphi(t) \\
\lambda\left(t, \bar{S}_{t}^{*}\right) & =\frac{\left(\bar{S}_{t}^{*}\right)^{2(1-a)}}{\Delta \varphi(t)}, \quad x(t)=\frac{(K)^{2(1-a)}}{\Delta \varphi(t)} .
\end{aligned}
$$

In the following, we use $\chi_{\nu}^{2}$ to denote a chi-square distributed random variable with $\nu$ degrees of freedom and $\chi_{\nu}^{2}(\lambda)$ to denote a non-central chi-square distributed random variable with $\nu$ degrees of freedom and non-centrality parameter $\lambda$. The probability density of $\chi_{\nu}^{2}(\lambda)$ is given by $p(\cdot, \nu, \lambda)$ and that of $\chi_{\nu}^{2}$ by $p(\cdot, \nu, 0)$. The corresponding cumulative distribution functions are denoted by $\Psi(x, \nu, \lambda)$ and $\Psi(x, \nu, 0):=$ $\int_{0}^{x} p(y, \nu, 0) d y$, respectively. We now present the fair price for a savings account bond, which pays one unit of the savings account at maturity.

Proposition 5.3 Under the TCEV model the discounted real-world price at time $t$ for a savings account bond, that is, for one unit of the savings account at maturity $T$, is given by the formula

$$
\begin{aligned}
\bar{Q}\left(t, T, \bar{S}_{t}^{*}\right) & :=\bar{S}_{t}^{*} E_{t}\left(\frac{1}{\bar{S}_{T}^{*}}\right) \\
& =\Psi\left(\lambda\left(t, \bar{S}_{t}^{*}\right), \frac{1}{1-a}, 0\right),
\end{aligned}
$$

and the corresponding fair strategy is characterized by 


$$
\begin{aligned}
\delta_{t}^{*} & =\frac{\partial \bar{Q}\left(t, T, \bar{S}_{t}^{*}\right)}{\partial \bar{S}_{t}^{*}}=p\left(\lambda\left(t, \bar{S}_{t}^{*}\right), \frac{1}{1-a}, 0\right) \frac{\partial \lambda\left(t, \bar{S}_{t}^{*}\right)}{\partial \bar{S}_{t}^{*}} \\
\delta_{t}^{0} & =\bar{Q}\left(t, T, \bar{S}_{t}^{*}\right)-\delta_{t}^{*} \bar{S}_{t}^{*}
\end{aligned}
$$

where

$$
\frac{\partial \lambda\left(t, \bar{S}_{t}^{*}\right)}{\partial \bar{S}_{t}^{*}}=2(1-a) \frac{\left(\bar{S}_{t}^{*}\right)^{1-2 a}}{\Delta \varphi(t)} .
$$

Some readers might be surprised by the result presented in Proposition 5.3, and may instead have expected the discounted savings account bond price process to be

$$
\tilde{\bar{Q}}\left(t, T, \bar{S}_{t}^{*}\right)=1
$$

and

$$
\tilde{\delta}_{t}^{0}=1 \quad, \tilde{\delta}_{t}^{*}=0, \quad t \in[0, T],
$$

which means that at time 0 , one unit of the savings account is purchased and kept until maturity. This strategy clearly replicates the claim and is also self-financing. However, it is not a fair portfolio, since the associated benchmarked portfolio is a strict supermartingale and not a martingale, as the next result shows, which follows directly from Proposition 5.1 and the observation that $\Lambda_{t}=1 / \bar{S}_{t}^{*}=\hat{B}_{t}$, for all $t \geq 0$.

Proposition 5.4 The above benchmarked portfolio $\hat{S}_{t}^{\tilde{\delta}}:=\frac{S_{t}^{\tilde{\delta}}}{S_{t}^{*}}$, where $\tilde{\delta}=\left\{\tilde{\delta}_{t}=\right.$ $\left.\left(\tilde{\delta}_{t}^{0}, \tilde{\delta}_{t}^{1}\right), t \geq 0\right\}$, is a strict supermartingale, i.e.,

$$
\hat{S}_{t}^{\tilde{\delta}}>E_{t}\left(\hat{S}_{u}^{\tilde{\delta}}\right)
$$

where $0 \leq t<u<\infty$.

Hence Proposition 5.4 considers a portfolio which is not fair. It simply holds one unit of the savings account and replicates the payoff using a self-financing strategy, but not at the minimal price. The discounted minimal price is given in Proposition 5.3 as the probability of a chi-square distributed random variable. This probability is less than the constant one, representing the discounted price of one unit of the savings account.

We now present the fair price of a call option. In our case, the strike price of the call option is chosen to be $K B_{T}$, where $K$ is a fixed positive constant. ${ }^{3}$ From an economic point of view, it is plausible to index the strike price with the savings account, as it compares the performance of the GOP to the savings account. In particular, for longdated derivatives, this feature is attractive, as the probability of the GOP finishing below a fixed level decreases as time to maturity increases.

Proposition 5.5 The discounted real-world price at time $t$ of a European call option

3 One can also derive explicit pricing formulas for call and put options with strike price $K$. However, this would require us to make an explicit assumption regarding the short rate dynamics. The presented pricing problem avoids such an assumption. 
with strike $K B_{T}$ and maturity $T$ is given by

$$
\begin{aligned}
\bar{C}\left(t, T, \bar{S}_{t}^{*}\right) & :=\bar{S}_{t}^{*} E_{t}\left(\frac{\left(\bar{S}_{T}^{*}-K\right)^{+}}{\bar{S}_{T}^{*}}\right) \\
& =\bar{S}_{t}^{*}\left(1-\Psi\left(x(t), \nu, \lambda\left(t, \bar{S}_{t}^{*}\right)\right)\right)-K \Psi\left(\lambda\left(t, \bar{S}_{t}^{*}\right), \frac{1}{1-a}, x(t)\right),
\end{aligned}
$$

where $\nu=\frac{3-2 a}{1-a}, x(t)$ is defined in (5.5) and the fair strategy is given by

$$
\begin{aligned}
\delta_{t}^{*}= & \left(1-\Psi\left(x(t), \nu, \lambda\left(t, \bar{S}_{t}^{*}\right)\right)\right) \\
& -\frac{\bar{S}_{t}^{*}}{2}\left(\Psi\left(x(t), \nu+2, \lambda\left(t, \bar{S}_{t}^{*}\right)\right)-\Psi\left(x(t), \nu, \lambda\left(t, \bar{S}_{t}^{*}\right)\right)\right) \frac{\partial \lambda\left(t, \bar{S}_{t}^{*}\right)}{\partial \bar{S}_{t}^{*}} \\
& -K p\left(\lambda\left(t, \bar{S}_{t}^{*}\right), \frac{1}{1-a}, x(t)\right) \frac{\partial \lambda\left(t, \bar{S}_{t}^{*}\right)}{\partial \bar{S}_{t}^{*}}
\end{aligned}
$$

and

$$
\delta_{t}^{0}=\bar{C}\left(t, T, \bar{S}_{t}^{*}\right)-\delta_{t}^{*} \bar{S}_{t}^{*}
$$

We conclude this section by presenting the fair price for put options. Again, we choose to index the strike price with the savings account.

Proposition 5.6 The discounted real-world price at time $t$ of a European put option with strike $K B_{T}$ and maturity $T$ is given by

$$
\begin{aligned}
\bar{F}\left(t, T, \bar{S}_{t}^{*}\right):= & \bar{S}_{t}^{*} E_{t}\left(\frac{\left(K-\bar{S}_{T}^{*}\right)^{+}}{\bar{S}_{T}^{*}}\right) \\
= & K\left(\Psi\left(\lambda\left(t, \bar{S}_{t}^{*}\right), \frac{1}{1-a}, 0\right)-\Psi\left(\lambda\left(t, \bar{S}_{t}^{*}\right), \frac{1}{1-a}, x(t)\right)\right) \\
& -\bar{S}_{t}^{*} \Psi\left(x(t), \nu, \lambda\left(t, \bar{S}_{t}^{*}\right)\right),
\end{aligned}
$$

where $x(t)$ is defined in (5.5). The fair strategy is given by

$$
\begin{aligned}
\delta_{t}^{*}= & K\left(p\left(\lambda\left(t, \bar{S}_{t}^{*}\right), \frac{1}{1-a}, 0\right)-p\left(\lambda\left(t, \bar{S}_{t}^{*}\right), \frac{1}{1-a}, x(t)\right)\right) \frac{\partial \lambda\left(t, \bar{S}_{t}^{*}\right)}{\partial \bar{S}_{t}^{*}} \\
& -\left(\Psi\left(x(t), \frac{3-2 a}{1-a}, \lambda\left(t, \bar{S}_{t}^{*}\right)\right)\right. \\
& \left.+\frac{\bar{S}_{t}^{*}}{2}\left(\Psi\left(x(t), \frac{1}{1-a}+4, \lambda\left(t, \bar{S}_{t}^{*}\right)\right)-\Psi\left(x(t), \frac{3-2 a}{1-a}, \lambda\left(t, \bar{S}_{t}^{*}\right)\right)\right) \frac{\partial \lambda\left(t, \bar{S}_{t}^{*}\right)}{\partial \bar{S}_{t}^{*}}\right)
\end{aligned}
$$

and

$$
\delta_{t}^{0}=\bar{F}\left(t, T, \bar{S}_{t}^{*}\right)-\delta_{t}^{*} \bar{S}_{t}^{*}
$$


Finally, we reassure the reader that under the benchmark approach, the well-known put-call parity holds, when savings account bond, call and put prices are computed using Propositions 5.3, 5.5, and 5.6, respectively, see equation (13.2.20) in Platen and Heath (2010).

\section{Empirical Analysis}

In this section, we discuss the performance of the hedging strategies presented in Section 5 using two examples. The first example demonstrates how savings account bonds can be hedged by means of dynamic trading in savings account and GOP. The second example illustrates the hedging of a European put option using savings account and GOP. ${ }^{4}$ In both examples pricing and hedging is performed for the MMM and the TCEV model, using the parameter estimates obtained in Section 4 and the formulae derived in Section 5. This will allow us to study the improvements made possible by the richer TCEV model.

We begin with the first example, illustrating daily hedging of a savings account bond by means of a savings account and the EWI114, which is used to approximate the GOP. As motivated above, since the results presented in the previous section assume that the hedging portfolio is rebalanced continuously, it seems to be relevant to employ daily data, rather than monthly data. ${ }^{5}$ We plot the self-financing hedge portfolios for savings account bonds, starting on 01 January 1973 and maturing in July 2010, under daily reallocation in the left panel of Figure 4. We observe that the MMM produces a more expensive hedge than the TCEV model. In addition, the benchmarked hedge portfolios are plotted in the right panel of Figure 4. One observes from the figure that in the case of the TCEV model specification the underlying assumption regarding the benchmarked portfolio to be a martingale, see Definition 5.1, seems to be reasonably satisfied, whereas in the case of the MMM one may have to say that the benchmarked hedge portfolio is more likely to be a strict supermartingale. This suggests that the extra degrees of freedom offered by the TCEV are important to ensure that Definition 5.1 is better satisfied.

Every day, the fraction of wealth $\pi_{t}^{*}$ invested in the EWI114 is adjusted in a selffinancing manner according to the value of $\delta_{t}^{*}$ computed for the model under consideration. In the left panel of Figure 5 we observe that the TCEV model invests initially almost the total amount of wealth in the GOP. Furthermore, the TCEV model invests a larger fraction of wealth in the GOP, compared to the MMM. We note that as time to maturity decreases, a larger portion of wealth is invested in the savings account, which is in line with Samuelson (1979) who argues that one should not use the GOP as the only investment for short term horizons.

4 We have also studied the hedging of call options, but omit the results from the paper since the hedge performance is similar to that of a put.

5 Hedging performance has also been tested using diversified indices on a monthly basis, such as, e.g., the S\&P 500. The results are qualitatively similar to those obtained using daily data for the EWI114 as GOP and thus, are not presented in the paper. 

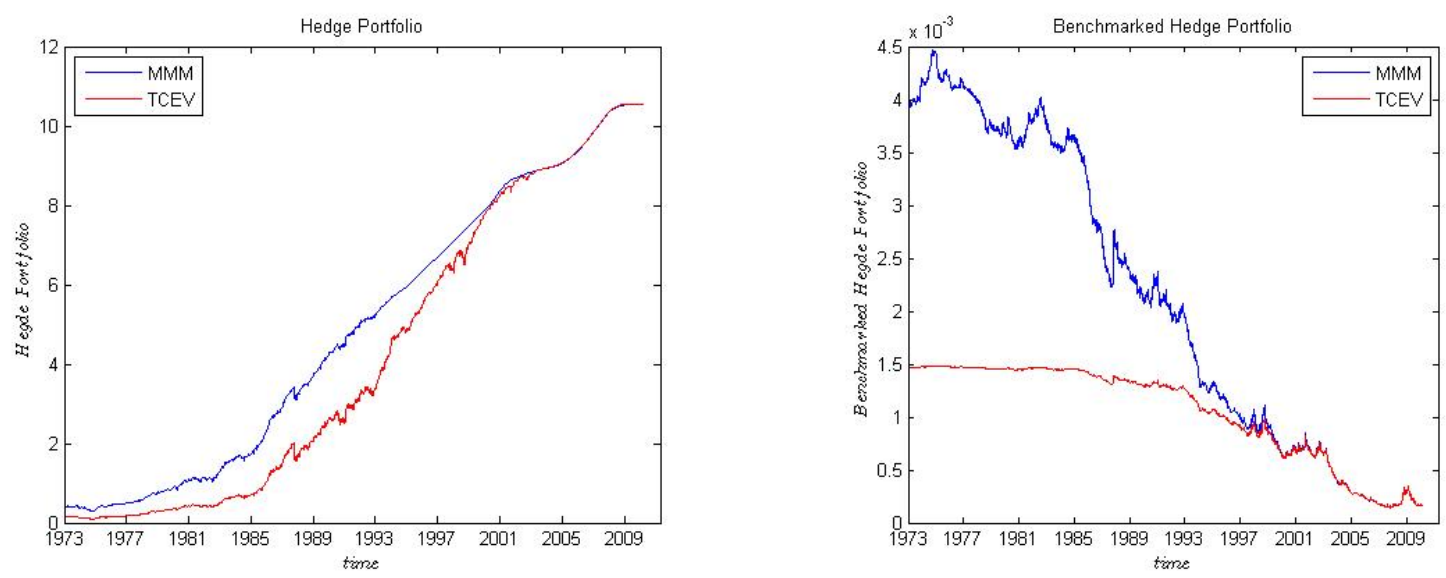

Fig. 4. Hedge portfolio (left panel) and benchmarked hedge portfolio (right panel) for MMM and TCEV model specifications for daily hedging of a savings account.
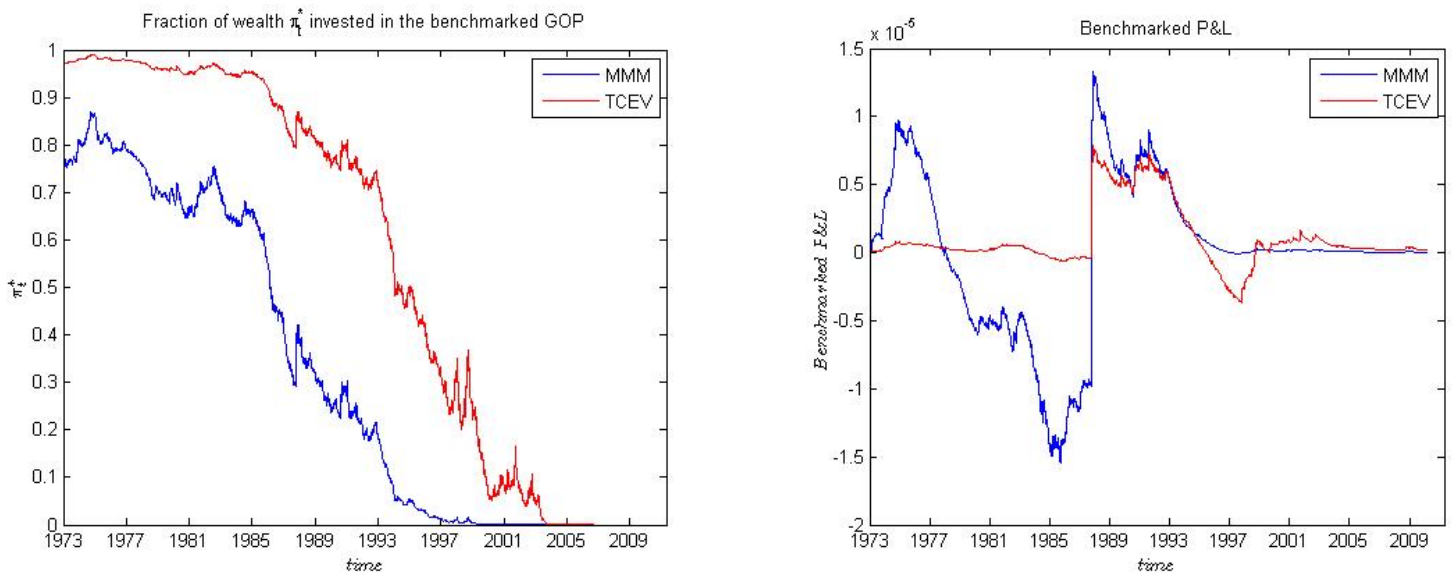

Fig. 5. Left Panel: Estimated fraction of wealth $\pi_{t}^{*}$ invested in the GOP for daily hedging of a savings account. Right Panel: benchmarked profit and loss (P\&L).

When defining the benchmarked profit and loss (P\&L) as the benchmarked price minus benchmarked hedge portfolio, the MMM and the TCEV model result in benchmarked P\&Ls that are both rather small in absolute value. These are visualized in the right panel of Figure 5. The maximum absolute benchmarked P\&L amounts to less than 0.000015 for both model specifications. Moreover, it can be seen from the figure that the benchmarked P\&L fluctuates significantly during the recession periods, such as the Wall Street crash in 1987 and the dot-com bubble in 1995-2000. Comparing the model specifications, one observes that the TCEV model outperforms the MMM in terms of minimal price and comparable benchmarked P\&L. Furthermore, the TCEV model is more robust in recession periods, compared to the MMM. In fact, the TCEV model results are hardly affected by the equity market meltdown starting from the crisis in 1987, which is due to the fact that a large fraction of wealth $\pi_{t}^{*}$ is at that time invested in the GOP. Overall, we observe that the TCEV model provides the more cost-efficient hedge and leads to a smaller benchmarked P\&L than the MMM.

The second example illustrates daily hedging of a put option whereby the EWI114 is used as a proxy for the GOP. As in the previous example, a self-financing hedge 

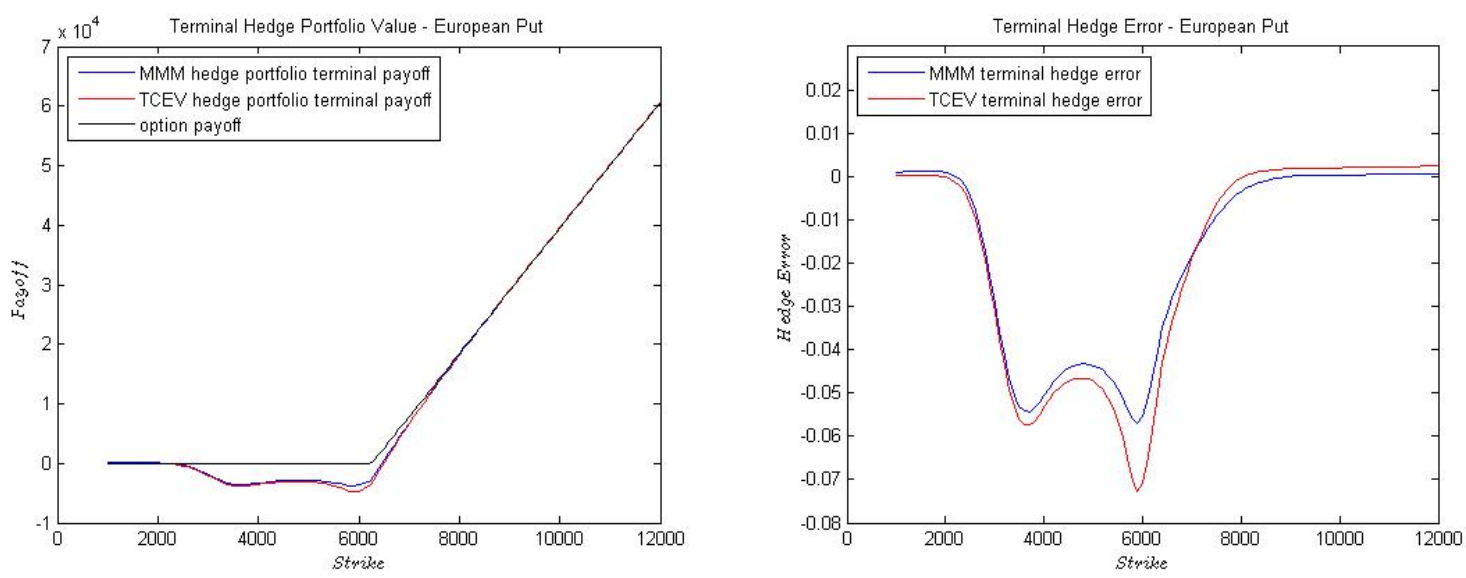

Fig. 6. Terminal hedge portfolio value (left panel) and terminal hedge error (right panel) for the MMM and the TCEV model specifications for a daily hedging of a put option.

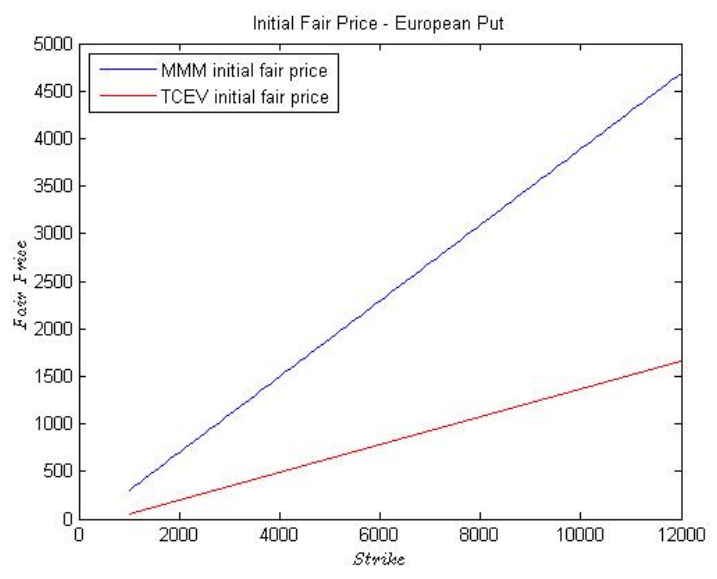

Fig. 7. Initial fair price for the MMM and the TCEV model specifications for a daily hedging of a put option.

portfolio reallocates daily $\delta_{t}^{*}$ units in the GOP, and $\delta_{t}^{0}$ units in the savings account. The left panel of Figure 6 shows the terminal hedge portfolio for the put option plotted against different strikes. The hedge performance appears to be roughly similar for both model specifications and quite good when the put option is in-the-money. It worsens for the at- and out-of-the-money case. Whenever strikes are close to the terminal index value, that is, when the option is at-the-money, the hedge performance is the worst. In fact, this is most likely a consequence of the large negative gamma of at-the-money options, which causes, in general, larger hedge errors for infrequent delta hedging. The right panel of Figure 6 refines the picture, by plotting terminal hedge errors against strikes for both models. We observe again that close to the atthe-money strike one has higher errors, which is an established stylized feature of discrete hedging, and not indicative of model misspecification. When plotting the initial fair price for both model specifications in Figure 7, we observe that the less expensive hedge is achieved by the TCEV model specification. We conclude that the TCEV model provides the more cost-efficient hedge compared to the MMM, while generating a comparable hedge error and, thus, a comparable P\&L. 

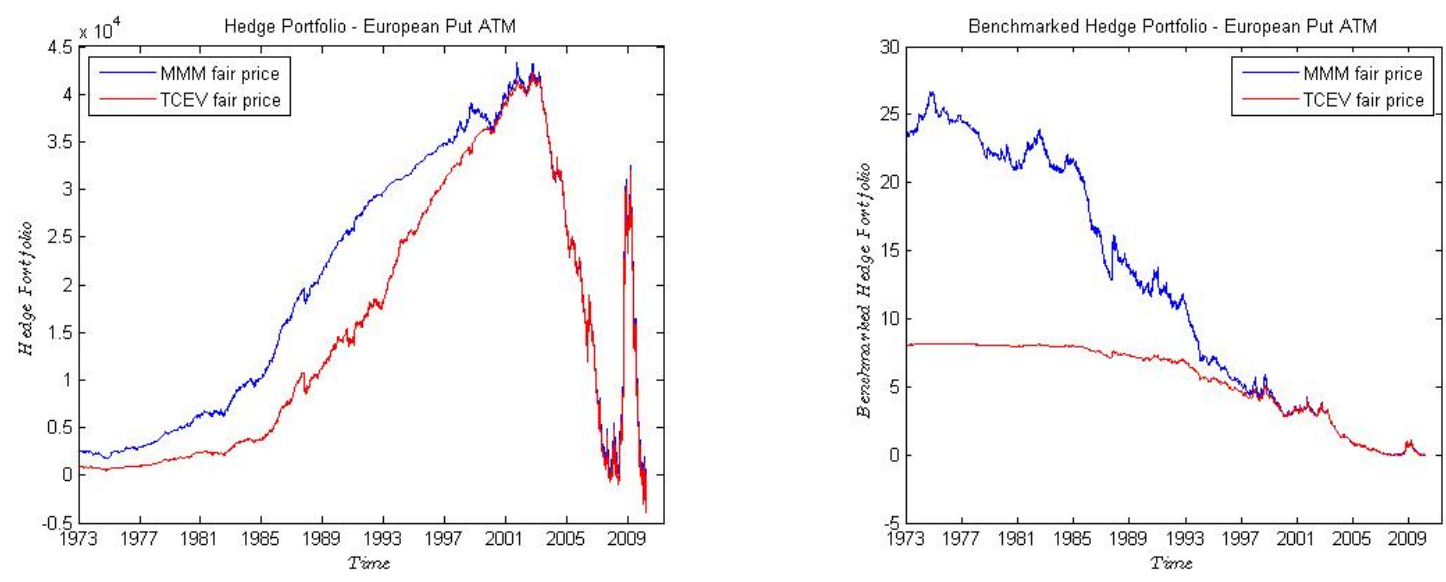

Fig. 8. Hedge portfolio (left panel) and benchmarked hedge portfolio (right panel) as a function of time for MMM and TCEV model specifications for a daily hedge of a put option and strikes close to at-the-money.

To confirm our findings regarding the hedging costs, the left panel of Figure 8 shows the evolution of the hedge portfolios over time for the MMM and the TCEV model specifications and the at-the-money (ATM) strike. ${ }^{6}$ We observe now over time how the MMM provides the most expensive hedge, followed by the TCEV model. Furthermore, the benchmarked hedge portfolio plotted in the right panel of Figure 8 confirms that the underlying assumption, regarding the martingale property of the benchmarked hedge portfolio, is more likely fulfilled under the TCEV model.

Furthermore, we observe that the hedge portfolios for both model specifications assume similar values as the maturity date of the option approaches. It is interesting to observe in Figure 8 the large draw down in the value of the hedge portfolio, followed by the rapid increase around the period of the financial crisis. In fact, a put option has a negative exposure to the GOP and thus, an increase in the GOP will lead to a decline in the value of the hedge portfolio and vice versa, compare also Figure 1 with Figure 8 to note this fact.

\section{Conclusion}

We have shown that for a time-dependent variant of a constant elasticity of variance model, the TCEV model, the necessary parameters can be successfully estimated. The model can be interpreted as a generalization of the stylized version of the minimal market model (MMM), a model which has been extensively used to model the growth optimal portfolio. The TCEV model retains a key advantage of the MMM, namely one can still derive closed-form solutions for key financial products, such as savings account bonds, as well as, call and put options. Furthermore, the model is easily fitted using techniques from non-parametric kernel-based estimation. Finally, we find that

$\overline{6}$ Results for in-the-money and out-of-the-money options are similar, and thus, are not presented here. 
the TCEV model outperforms the stylized version of the MMM with respect to the pricing and hedging of some standard derivatives.

We envisage that the TCEV model can be used to reduce the prices of financial derivatives, in particular long-dated claims, as they occur for example in pension guarantees or variable annuities.

\section{Appendix}

In this appendix, we discuss probabilistic aspects of the TCEV model, which we used to prove the results presented in Sections 2 and 5 .

We work on a filtered probability space $(\Omega, \mathcal{A}, \mathcal{A}, P)$, which carries one source of uncertainty $W=\left\{W_{t}, t \geq 0\right\}$, a standard Wiener process. The discounted GOP $\bar{S}^{*}=\left\{\bar{S}_{t}^{*}, t \geq 0\right\}$, satisfies the following stochastic differential equation

$$
d \bar{S}_{t}^{*}=\bar{S}_{t}^{*}\left(c^{2} \alpha_{t}^{2-2 a}\left(\bar{S}_{t}^{*}\right)^{2 a-2} d t+c \alpha_{t}^{1-a}\left(\bar{S}_{t}^{*}\right)^{a-1} d W_{t}\right) .
$$

The following approach closely follows Carr and Linetsky (2006) and Hulley (2009), in particular, we show that the discounted GOP can be represented as a time-changed squared Bessel process. This result is key to the approach presented in this appendix, and subsequent results follow easily from it. Recall from (2.14) the notation

$$
\varphi(t)=\frac{(1-a) \alpha_{0}^{2-2 a} c^{2}(\exp \{2(1-a) \eta t\}-1)}{2 \eta} .
$$

Proposition 8.1 The process $\bar{S}^{*}=\left\{\bar{S}_{t}^{*}, t \geq 0\right\}$ satisfies the following equality in distribution

$$
\bar{S}_{t}^{*} \stackrel{(d)}{=} Z_{\varphi(t)}^{\frac{1}{2-2 a}}
$$

where $Z$ is a squared Bessel process of dimension $\nu=\frac{3-2 a}{1-a}$.

Proof. Define the process $X=\left\{X_{t}, t \geq 0\right\}$ via $X_{t}=\alpha_{0}^{2-2 a} Y_{t}^{2-2 a}$, then

$$
d X_{t}=\left(\alpha_{0}^{2-2 a} c^{2}(1-a)(3-2 a)-\eta X_{t} 2(1-a)\right) d t+2(1-a) \alpha_{0}^{1-a} c \sqrt{X_{t}} d W_{t} .
$$

Hence $X$ is a square-root process, and it follows from Pitman and Yor (1982) and Platen and Heath (2010) that

$$
X_{t} \stackrel{(d)}{=} \exp \{-2(1-a) \eta t\} Z_{\varphi(t)},
$$

which completes the proof.

We now investigate whether the TCEV model admits an equivalent martingale measure (EMM); recalling from Platen and Heath (2010) that the MMM, a special case of the TCEV, does not admit an EMM, this question seems worthwhile. We define 
the candidate Radon-Nikodym derivative process $\Lambda=\left\{\Lambda_{t}, t \in[0, T]\right\}$, via

$$
\Lambda_{t}=\frac{\hat{B}_{t}}{\hat{B}_{0}}=\frac{\bar{S}_{0}^{*}}{\bar{S}_{t}^{*}}=\frac{\alpha_{0} Y_{0}}{\alpha_{t} Y_{t}}
$$

for a given fixed $T$. If $\Lambda=\left\{\Lambda_{t}, t \in[0, T]\right\}$ is a martingale, then we can use $\Lambda_{T}$ to define an EMM, via the Girsanov theorem. However, recall from the discussion in Section 5, that $\Lambda=\left\{\Lambda_{t}, t \in[0, T]\right\}$ is a supermartingale. Hence we need to investigate if it is a strict supermartingale, or not. The proof is completed by making use of Proposition 8.1 , and the following well-known properties of the non-central $\chi^{2}$-distribution, see Johnson et al. (1994):

Lemma 8.1 The following relations hold:

$$
\frac{Z_{\varphi(t)}}{\varphi(t)} \sim \chi_{\nu}^{2}\left(\frac{x}{\varphi(t)}\right)
$$

where $Z$ is a squared Bessel process of dimension $\nu>2$ with $Z_{\varphi(0)}=x$. Furthermore,

$$
\begin{aligned}
\left(\frac{\lambda}{x}\right)^{\frac{\nu-2}{2}} p(x, \nu, \lambda) & =p(\lambda, \nu, x) \\
\int_{0}^{\infty} p(x, \nu+2, y) d y & =\Psi(x, \nu, 0) \\
\int_{\lambda}^{\infty} p(x, \nu+2, y) d y & =\Psi(x, \nu, \lambda) \\
\int_{0}^{\lambda} p(x, \nu+2, y) d y & =\Psi(x, \nu, 0)-\Psi(x, \nu, \lambda) \\
\frac{\partial \Psi(x, \nu, \lambda)}{\partial \lambda} & =\frac{1}{2}(\Psi(x, \nu+2, \lambda)-\Psi(x, \nu, \lambda)) .
\end{aligned}
$$

We are now in a position to show that $\Lambda=\left\{\Lambda_{t}, t \in[0, T]\right\}$ is a strict supermartingale.

\section{Proof of Proposition 5.1:}

Recall from Section 5 that $\Lambda$ is a supermartingale. We now establish that it is a strict supermartingale by a straightforward calculation.

$$
\begin{aligned}
E\left(\Lambda_{T}\right) & =E\left(\frac{\alpha_{0} Y_{0}}{\alpha_{T} Y_{T}}\right)=E\left(\frac{\alpha_{0} Y_{0}}{Z_{\varphi(T)}^{\frac{1}{2-2 a}}}\right) \\
& =\frac{\alpha_{0} Y_{0}}{\varphi^{\frac{1}{2-2 a}}(T)} E\left(\frac{1}{\left(\chi_{\nu}^{2}\left(\lambda\left(0, \bar{S}_{0}^{*}\right)\right)\right)^{\frac{1}{2-2 a}}}\right)
\end{aligned}
$$




$$
\begin{aligned}
& =\frac{\alpha_{0} Y_{0}}{\varphi^{\frac{1}{2-2 a}}(T)} \int_{0}^{\infty}\left(\frac{\lambda\left(0, \bar{S}_{0}^{*}\right)}{y}\right)^{\frac{\nu-2}{2}} \frac{1}{\left(\lambda\left(0, \bar{S}_{0}^{*}\right)\right)^{\frac{\nu-2}{2}}} p\left(x, \nu, \lambda\left(0, \bar{S}_{0}^{*}\right)\right) d x \\
& =\int_{0}^{\infty} p\left(\lambda\left(0, \bar{S}_{0}^{*}\right), \nu, x\right) d x \\
& =\Psi\left(\lambda\left(0, \bar{S}_{0}^{*}\right), \frac{1}{1-a}, 0\right)<1=\Lambda_{0},
\end{aligned}
$$

where we used Proposition 8.1, and equations (8.3), (8.4), and (8.5).

We note that $\Lambda$ is in fact the only candidate for the Radon-Nikodym derivative of an EMM. This is due to the fact that we consider a two-asset market model consisting of the GOP $S_{t}^{*}$ and the savings account $B_{t}$. Since $S_{t}^{*}$ is the numéraire for the real world measure $P$, we only require to check if there is an EMM for which $B_{t}$ is a numéraire. The Radon-Nikodym derivative of such a measure is given by $\Lambda$, which is, as shown above, a strict local martingale and thus a strict supermartingale.

The above result means that risk-neutral pricing is not applicable, however, realworld pricing, in the sense of Platen and Heath (2010), still applies. We point out that Proposition 5.4 can be established using the same argument. In the following, we present pricing formulae for savings account zero coupon bonds, calls, and puts.

\section{Proof of Proposition 5.3:}

Using Proposition 8.1 and Lemma 8.1, we have

$$
\begin{aligned}
\bar{Q}\left(t, T, \bar{S}_{t}^{*}\right) & =\bar{S}_{t}^{*} E_{t}\left(\frac{1}{\bar{S}_{T}^{*}}\right)=\bar{S}_{t}^{*} E_{t}\left(\frac{1}{\alpha_{T} Y_{T}}\right)=\bar{S}_{t}^{*} E_{t}\left(\frac{1}{Z_{\varphi(T)}^{\frac{1}{2-2 a}}}\right) \\
& =\bar{S}_{t}^{*} \int_{0}^{\infty} \frac{1}{(\Delta \varphi(t) y)^{\frac{1}{2-2 a}}} p\left(y, \nu, \lambda\left(t, \bar{S}_{t}^{*}\right)\right) d y \\
& =\int_{0}^{\infty}\left(\left(\bar{S}_{t}^{*}\right)^{2(1-a)} \frac{1}{\Delta \varphi(t)}\right)^{\frac{1}{2-2 a}} \frac{1}{y^{\frac{1}{2-2 a}}} p\left(y, \nu, \lambda\left(t, \bar{S}_{t}^{*}\right)\right) d y \\
& =\int_{0}^{\infty}\left(\frac{\lambda\left(t, \bar{S}_{t}^{*}\right)}{y}\right)^{\frac{1}{2-2 a}} p\left(y, \nu, \lambda\left(t, \bar{S}_{t}^{*}\right)\right) d y \\
& =\int_{0}^{\infty} p\left(\lambda\left(t, \bar{S}_{t}^{*}\right), \nu, y\right) d y \\
& =\Psi\left(\lambda\left(t, \bar{S}_{t}^{*}\right), \frac{1}{1-a}, 0\right) .
\end{aligned}
$$

We now define the function

$$
u\left(t, \hat{B}_{t}\right)=\bar{Q}\left(t, T, \bar{S}_{t}^{*}\right) \hat{B}_{t}=\Psi\left(\lambda\left(t, \bar{S}_{t}^{*}\right), \frac{1}{1-a}, 0\right) \hat{B}_{t}
$$


and confirm that

$$
d u\left(t, \hat{B}_{t}\right)=\frac{\partial u\left(t, \hat{B}_{t}\right)}{\partial \hat{B}_{t}} d \hat{B}_{t}
$$

Hence

$$
\delta_{t}^{0}=\frac{\partial u\left(t, \hat{B}_{t}\right)}{\partial \hat{B}_{t}}
$$

Now, using

$$
\bar{Q}\left(t, T, \bar{S}_{t}^{*}\right)=\delta_{t}^{*} \bar{S}_{t}^{*}+\delta_{t}^{0},
$$

we obtain (5.6) and lastly (5.7).

For call options, we proceed in the same fashion.

\section{Proof of Proposition 5.5:}

Similarly to the proof of Proposition 5.3, we have

$$
\begin{aligned}
\bar{C}\left(t, T, \bar{S}_{t}^{*}\right) & =\bar{S}_{t}^{*} E_{t}\left(\frac{\left(\bar{S}_{T}^{*}-K\right)^{+}}{\bar{S}_{T}^{*}}\right) \\
& =\bar{S}_{t}^{*} E_{t}\left(\left(1-\left(\frac{x(t)}{\frac{Z_{\varphi(T)}}{\Delta \varphi(t)}}\right)^{\frac{1}{2-2 a}}\right)\right) \\
& =\bar{S}_{t}^{*} \int_{x(t)}^{\infty}\left(1-\left(\frac{x(t)}{y}\right)^{\frac{1}{2-2 a}}\right) p\left(y, \nu, \lambda\left(t, \bar{S}_{t}^{*}\right)\right) d y \\
& =\bar{S}_{t}^{*}\left(1-\Psi\left(x(t), \nu, \lambda\left(t, \bar{S}_{t}^{*}\right)\right)\right)-\bar{S}_{t}^{*} \int_{x(t)}^{\infty}\left(\frac{x(t)}{y}\right)^{\frac{1}{2-2 a}} p\left(y, \nu, \lambda\left(t, \bar{S}_{t}^{*}\right)\right) d y .
\end{aligned}
$$

However,

$$
\bar{S}_{t}^{*} \int_{x(t)}^{\infty}\left(\frac{x(t)}{y}\right)^{\frac{1}{2-2 a}} p\left(y, \nu, \lambda\left(t, \bar{S}_{t}^{*}\right)\right) d y=K \Psi\left(\lambda\left(t, \bar{S}_{t}^{*}\right), \frac{1}{1-a}, x(t)\right) .
$$

Setting

$u\left(t, \hat{B}_{t}\right):=\bar{C}\left(t, T, \bar{S}_{t}^{*}\right) \hat{B}_{t}=\left(1-\Psi\left(x(t), \nu, \lambda\left(t, \bar{S}_{t}^{*}\right)\right)\right)-K \Psi\left(\lambda\left(t, \bar{S}_{t}^{*}\right), \frac{1}{1-a}, x(t)\right) \hat{B}_{t}$,

we now have

$$
d u\left(t, \hat{B}_{t}\right)=\frac{\partial u\left(t, \hat{B}_{t}\right)}{\partial \hat{B}_{t}} d \hat{B}_{t},
$$

and we complete the proof in the same way as the proof of Proposition 5.3, in particular, using the the result in Lemma 8.1.

Lastly, we consider put options. 


\section{Proof of Proposition 5.6:}

Arguing as in the proofs of Propositions 5.3 and 5.5, we have

$$
\begin{aligned}
\bar{F}\left(t, T, \bar{S}_{t}^{*}\right)= & \bar{S}_{t}^{*} E_{t}\left(\frac{\left(K-\bar{S}_{T}^{*}\right)^{+}}{\bar{S}_{T}^{*}}\right) \\
= & \bar{S}_{t}^{*} E_{t}\left(\left(\frac{(x(t))^{\frac{1}{2-2 a}}}{\left(\frac{Z_{\varphi(T)}}{\Delta \varphi(t)}\right)^{\frac{1}{2-2 a}}}-1\right)\right) \\
= & \bar{S}_{t}^{*} \int_{0}^{x(t)}\left(\frac{x(t)}{y}\right)^{\frac{1}{2-2 a}} p\left(y, \nu, \lambda\left(t, \bar{S}_{t}^{*}\right)\right) d y-\bar{S}_{t}^{*} \int_{0}^{x(t)} p\left(y, \nu, \lambda\left(t, \bar{S}_{t}^{*}\right)\right) d y \\
= & \bar{S}_{t}^{*}\left(\frac{x(t)}{\lambda\left(t, \bar{S}_{t}^{*}\right)}\right)^{\frac{1}{2-2 a}} \int_{0}^{x(t)}\left(\frac{\lambda\left(t, \bar{S}_{t}^{*}\right)}{y}\right)^{\frac{1}{2-2 a}} p\left(y, \nu, \lambda\left(t, \bar{S}_{t}^{*}\right)\right) d y \\
& -\bar{S}_{t}^{*} \int_{0}^{x(t)} p\left(y, \nu, \lambda\left(t, \bar{S}_{t}^{*}\right)\right) d y \\
= & K\left(\Psi\left(\lambda\left(t, \bar{S}_{t}^{*}\right), \frac{1}{1-a}, 0\right)-\Psi\left(\lambda\left(t, \bar{S}_{t}^{*}\right), \frac{1}{1-a}, x(t)\right)\right) \\
& -\bar{S}_{t}^{*} \Psi\left(x(t), \nu, \lambda\left(t, \bar{S}_{t}^{*}\right)\right) .
\end{aligned}
$$

Again, setting

one can check that

$$
u\left(t, \hat{B}_{t}\right)=\bar{F}\left(t, T, \bar{S}_{t}^{*}\right) \hat{B}_{t},
$$

$$
d u\left(t, \hat{B}_{t}\right)=\frac{\partial u\left(t, \hat{B}_{t}\right)}{\partial \hat{B}_{t}} d \hat{B}_{t},
$$

and complete the proof in the same way as the proof of Proposition 5.3, in particular using the result in Lemma 8.1. 


\section{References}

Becherer, D., 2001. The numeraire portfolio for unbounded semimartingales. Finance and Stochastics 5(3), 327-341.

Black, F., Scholes, M., 1973. The pricing of options and corporate liabilities. The Journal of Political Economy 81(3), 637-654.

Botev, Z., Grotowski, J. F., Kroese, D. P., 2010. Kernel density estimation via diffusion. The Annals of Statistics 38(5), 2916-2957.

Breiman, L., 1960. Investment policies for expanding business optimal in a long run sense. Naval Research Logistics Quarterly 7 (4), 647-651.

Brown, R., Schaefer, S., 1994a. Interest rate volatility and the shape of the term structure. Philosophical Transactions of the Royal Society of London A 347, 563576.

Brown, R., Schaefer, S., 1994b. The term structure of real interest rates and the Cox, Ingersoll, and Ross model. Journal of Financial Econometrics 35, 3-42.

Carr, P., Linetsky, V., 2006. A jump to default extended CEV model: an application of Bessel processes 10, 303-330.

Cox, J., 1975. Notes on option pricing I: constant elasticity of variance diffusions. Working paper, Stanford University unpublised.

Delbaen, F., Schachermayer, W., 1994. A general version of the fundamental theorem of asset pricing. Mathematische Annalen 300, 463-520.

Delbaen, F., Schachermayer, W., 1998. The fundamental theorem of asset pricing for unbounded stochastic processes. Mathematische Annalen 312, 215-250.

Derman, E., Kani, I., 1994. The volatility smile and its implied tree. Goldman Sachs Quantitative Strategies Research Notes.

Duffie, D., Gârleanu, N., 2001. Risk and valuation of collateralized debt obligations. Financial Analysts Journal 57, 41-59.

Dupire, B., 1994. Pricing with a smile. Risk Magazine 7, 18-20.

Epanechnikov, V., 1969. Nonparametric estimates of multivariate probability density. Theory of Probability and Applications 14, 153-158.

Florens-Zmirou, D., 1993. On estimating the diffusion coeffcient from discrete observations. Journal of Applied Probability 30(4), 790-804.

Fontana, C., Runggaldier, W., 2012. Diffusion-Based Models for Financial Markets Without Martingale Measures. Biagini, F., Richter, A. and Schlesinger, H. (eds.): Risk Measures and Attitudes, An LMU Excellent Symposium.

Heath, D., Platen, E., 2002. Consistent pricing and hedging for a modified constant elasticity of variance model. Quantitative Finance 2, 459-467.

Heston, S., 1997. A simple new formula for options with stochastic volatility. Working paper, University of St. Louis unpublised.

Hulley, H., 2009. Strict local martingales in continuous financial market models. Ph.D. thesis, UTS, Sydney.

Hulley, H., Platen, E., 2012. Hedging for the long run. Mathematics and Financial Economics 6(2), 105-124.

Hulley, H., Schweizer, M., 2010. M6 - On Minimal Market Models and Minimal Martingale Measures. Chiarella, C. and Novikov, A. (eds.): Contemporary Quantitative Finance: Essays in Honour of Eckhard Platen.

Ignatieva, K., Platen, E., 2012. Estimating the diffusion coefficient function for a diversified world stock index. Computational Statistics \& Data Analysis 56(6), $1333-1349$. 
Jacod, J., 2000. Non-parametric kernel estimation of the coeffcient of a diffusion. Scandinavian Journal of Statistics 27(1), 83-96.

Jiang, G., Knight, J., 1997. A nonparametric approach to the estimation of diffusion processes, with an application to a short-term interest rate model. Econometric Theory 13(7), 615-645.

Johnson, N., Kotz, S., Balakrishnan, N., 1994. Continuous univariate distributions, Vol. 1, 2nd Edition. John Wiley, New York.

Karatzas, I., Shreve, S. E., 1991. Brownian Motion and Stochastic Calculus, 2nd Edition. Springer.

Kardaras, C., 2010. Finitely Additive Probabilities and the Fundamental Theorem of Asset Pricing. Chiarella, C. and Novikov, A. (eds.): Contemporary Quantitative Finance: Essays in Honour of Eckhard Platen.

Kelly, J. R., 1956. A new interpretation of information rate. The Bell Journal of Economics and Management Science 35, 917-926.

Künsch, H. R., 1989. The jackknife and the bootstrap for general stationary observations. The Annals of Statistics 17, 1217-1241.

Latané, H., 1959. Criteria for choice among risky ventures. Journal of Political Economy $38,145-155$.

Lewis, A., 2000. Option Valuation under Stochastic Volatility. Finance Press.

Loewenstein, M., Willard, G., 2000. Local martingales, arbitrage, and viability: Free snacks and cheap thrills. Econometric Theory 16(1), 135-161.

Long, J. B., 1990. The numeraire portfolio. Journal of Financial Economics 26, 29-69.

MacLean, L., Thorp, E., Ziemba, W., 2011. The Kelly Capital Growth Investment Criterion. World Scientific Handbook in Financial Economic Series, Vol. 3.

Markowitz, H., 1976. Investment for the long run: new evidence for an old rule. Journal of Finance XXXI (5), 1273-1286.

Merton, R., 1973. Theory of rational option pricing. The Bell Journal of Economics and Management Science 4, 141-183.

Pitman, J., Yor, M., 1982. A Decomposition of Bessel bridges. Zeitschrift für Wahrscheinlichkeitstheorie und Verwandte Gebiete 59 (4), 425-457.

Platen, E., 1997. A non-linear stochstic volatility model. Working paper, Australian National University unpublised.

Platen, E., 2001. A Minimal Financial Market Model. Birkhauser Verlag.

Platen, E., 2002. Arbitrage in continuous complete markets. Advances in Applied Probability 34(3), 540-558.

Platen, E., 2005. On the role of the growth optimal portfolio in finance. Australian Economic Papers, Blackwell Publishing 44(4), 365-388.

Platen, E., Heath, D., 2010. A Benchmark Approach to Quantitative Finance. Springer-Verlag.

Platen, E., Rendek, R., 2011. Approximating the numeraire portfolio by naive diversification. Journal of Asset Management 13(1), 34-50.

Revuz, D., Yor, M., 1999. Continous Martingales and Brownian Motion. Springer.

Samuelson, P. A., 1979. Why we should not make mean log of wealth big though years to act are long. Journal of Banking and Finance 3, 305-307.

Schroder, M., 1989. Computing the constant elasticity of variance option pricing formula. Journal of Finance 44(1), 211-219.

Scott, D., 1992. Multivariate density estimation: theory, practice and visualization. John Wiley, New York.

Silverman, B. W., 1986. Density Estimation. Chapman and Hall.

Soulier, P., 1998. Nonparametric estimation of the diffusion coeffcient of a diffusion 
process. Stochastic Analysis and Applications 16, 185-200.

Stanton, R., 1997. A nonparametric model of term structure dynamics and the market price of interest rate risk. Journal of Finance 52(5), 1973-2002.

Thorp, E. O., 1961. A favourable strategy for twenty-one. In: Proceedings of the National Academy of Sciences. Vol. 47. pp. 110-112.

Wand, M. P., Jones, M. C., 1995. Kernel Smoothing. Chapman and Hall. 